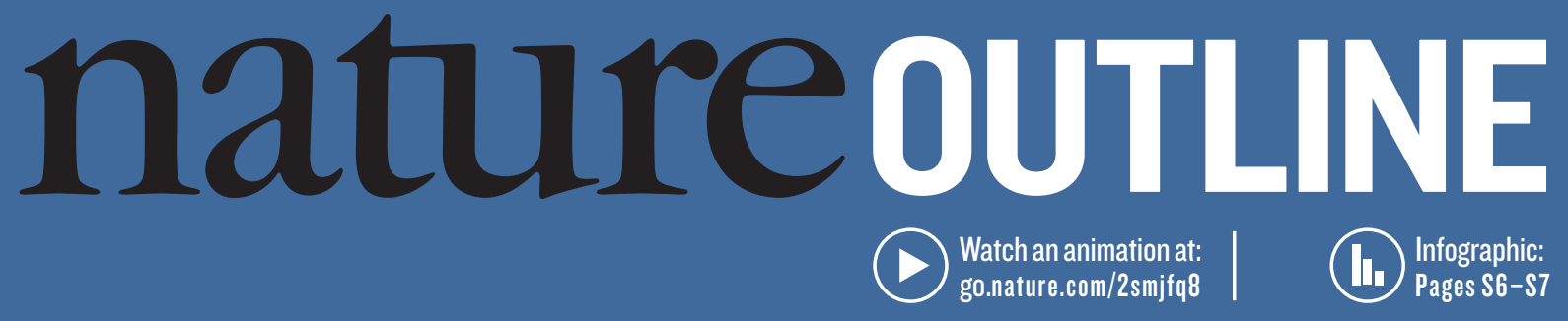

\title{
EARDRUM REGENERATION: MEMBRANE REPAIR
}

\section{Can tissue engineering provide a cheap and convenient alternative to surgery for eardrum repair?}

T he eardrum, or tympanic membrane, forms the interface between the outside world and the delicate bony structures of the middle ear - the ossicles - that conduct sound vibrations to the inner ear. At just a fraction of a millimetre thick and held under tension, the membrane is perfectly adapted to transmit even the faintest of vibrations. But the qualities that make the eardrum such a good conductor of sound come at a price: fragility. Burst eardrums are a major cause of conductive hearing loss - when sounds can't pass from the outer to the inner ear.

Most burst eardrums are caused by infections or trauma. The vast majority heal on their own in about ten days, but for a small proportion of people the perforation fails to heal naturally. These chronic ruptures cause conductive hearing loss and increase the risk of middle ear infections, which can have serious complications.

Surgical intervention is the only option for people with eardrums that won't heal. Tympanoplasty involves collecting graft material from the patient to use as a patch over the perforation. Tympanoplasty has a very good success rate but, as with any specialized surgical procedure, it doesn't come cheap, it requires specialist inpatient care and it carries a small risk of complications such as nerve damage as well as the side effects of general anaesthesia. The surgery is almost non-existent in many developing countries that, owing to higher rates of infection, are likely to have a higher prevalence of tympanic membrane perforations (T. S. Ibekwe et al. Otol. Neurotol. 28, 348-352; 2007).

It is clear that a more-convenient and less-expensive procedure would be a benefit, and tissue engineering looks increasingly as if it can deliver on both fronts. This Outline focuses on a technique developed by researchers in Japan that uses a gelatin sponge scaffold infused with basic fibroblast growth factor (bFGF) to help the eardrum regenerate without the need for a graft.

In a phase II study published in 2011, 52 out of 53 patients who received the procedure showed complete healing and improved hearing, compared with only 1 in 10 in the control

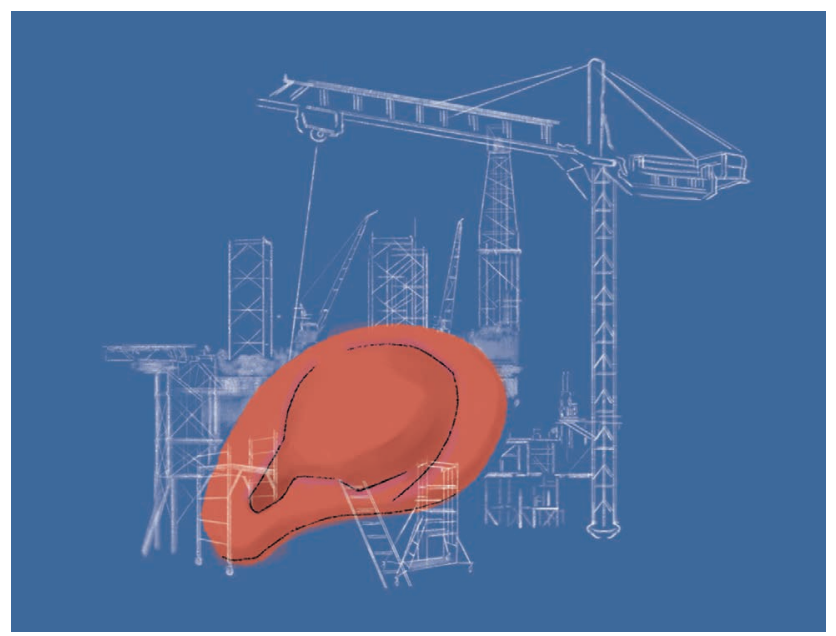

group (S. Kanemaru et al. Otol. Neurotol. 32, 1218-1223; 2011). In a commentary in the same journal, Robert Jackler, a head and neck surgeon at Stanford University, California, wrote that, should the results be replicated, the procedure represents "potentially the greatest advance in otology since the invention of the cochlear implant" (R. Jackler Otol. Neurotol. 33, 289; 2012). Not only would it replace many thousands of surgical procedures each year, Jackler speculated that "it could bring a simple and inexpensive remedy to the many millions of patients around the world for whom capital intensive microsurgery is not available".

Confirmation should come when results are published from a phase III trial that wrapped up in July 2016. Meanwhile, similar procedures in other areas of surgery have added further proof that the concept works. Preclinical studies have indicated that the combination of a gelatin scaffold and bFGF can aid healing of skin grafts and of scarred vocal chords; other growth factors, such as epidermal growth factor, are also being explored.

Nature is pleased to acknowledge the financial support of the Translational Research Informatics Center (TRI) and Kitano Hospital. As always, Nature retains sole responsibility for all editorial content. -

\section{David Holmes is a science writer in the United Kingdom.}
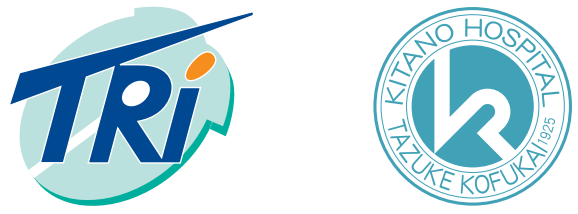